University of Nebraska - Lincoln

DigitalCommons@University of Nebraska - Lincoln

2012

\title{
Gender, Gender Roles, and Anxiety: Perceived Confirmability of Self Report, Behavioral Avoidance, and Physiological Reactivity
}

\author{
Milena Stoyanova \\ University of Nebraska-Lincoln \\ Debra Anne Hope \\ University of Nebraska-Lincoln, dhope1@unl.edu
}

Follow this and additional works at: https://digitalcommons.unl.edu/psychfacpub

Part of the Psychiatry and Psychology Commons

Stoyanova, Milena and Hope, Debra Anne, "Gender, Gender Roles, and Anxiety: Perceived Confirmability of Self Report, Behavioral Avoidance, and Physiological Reactivity" (2012). Faculty Publications, Department of Psychology. 570.

https://digitalcommons.unl.edu/psychfacpub/570

This Article is brought to you for free and open access by the Psychology, Department of at DigitalCommons@University of Nebraska - Lincoln. It has been accepted for inclusion in Faculty Publications, Department of Psychology by an authorized administrator of DigitalCommons@University of Nebraska - Lincoln. 


\title{
Gender, Gender Roles, and Anxiety: Perceived Confirmability of Self Report, Be- havioral Avoidance, and Physiological Reactivity
}

\author{
Milena Stoyanova and Debra A. Hope \\ University of Nebraska-Lincoln, Lincoln, Nebraska, United States
}

Keywords: Anxiety, Gender, Gender role, Behavioral avoidance, Physiological reactivity

\begin{abstract}
Despite the well-documented gender effect in anxiety, less is known about contributing factors to women's greater risk for anxiety and fears. The present study examined the relationship between gender, gender role orientation (i.e., expressivity/instrumentality) and fear of harmless insects (tarantula), using a multimodal approach of self-report measures, a Behavioral Approach Test (BAT), and physiological reactivity. Participants (144 college students; 67 women, 77 men) completed a questionnaire packet and then were instructed to approach a tarantula. We were unable to replicate Pierce and Kirkpatrick's (1992) findings that men underreport anxiety. Consistent with the literature, women in the study experienced greater anxiety and avoidance compared to men. However, men and women did not differ on physiological reactivity during the first $2 \mathrm{~min}$ of the BAT. The concordance across avoidance, anxiety and heart rate reactivity differed by gender, suggesting that men and women have different experiences when faced with a fearful object. Furthermore, instrumentality (masculinity) was negatively related to anticipatory anxiety for women but not for men.
\end{abstract}

Portions of this paper have been presented at the annual meetings of the Association for Behavioral and Cognitive Therapy in Orlando, Florida, 2008, and San Francisco, California, 2010.

Corresponding author: dhope1@unl.edu.

Article history: Received February 7, 2011; received in revised form November 6, 2011; accepted November 7, 2011.

\section{Introduction}

Research has consistently demonstrated that women tend to be at a greater risk for developing anxiety disorders than men (Bourdon et al., 1988; Weissman \& Marikangas, 1986). Data from large epidemiological studies indicate a gender ratio of 2:1 (women:men) for anxiety disorders (Angst \& Dobler-Mikola, 1985; Bruce et al., 2005). Using data from the National Comorbidity Study, Kessler, Chiu, Demler, Merikangas, and Walters (2005) found lifetime prevalence rates of $36.4 \%$ for women and $25.4 \%$ for men for anxiety disorders. Furthermore, several cross-cultural studies have reported a gender effect in fear reporting towards harmless animals or disgust-relevant animals, such as spiders, snakes, or worms (Davey et al., 1998). It appears that the extent of this gender effect varies according to specific types of stimuli, with fears of harmless animals (e.g., dogs, spiders) being most pronounced (Arrindell et al., 2003), whereas no gender effect was observed in fears of enclosed spaces, loud noise, or bodily injury (e.g., Tucker \& Bond, 1997). Davey et al. (1998) found that women were more fearful of disgust-relevant animals (e.g., worms, spiders) than men, whereas there was no difference in reported fear towards threat-posing animals (e.g., sharks, lions).

Despite these findings and the growing body of literature confirming the well-established gender effect, little is known about specific factors that may predispose women to this higher risk. Most researchers reporting a gender effect in anxiety often explain their findings by referring to well known prevalence rates or simply fail to provide an explanation. A recent review by McLean and Anderson (2009) concluded that different socialization experiences, which teach gender-specific expression and acceptable coping styles, might contribute to the gender effect in anxiety and fear.

In the literature, the terms gender and sex are often used interchangeably. However, since physiology is not the only factor that separates men from women, it is important to consider other differences such as cultural, economic, and social variations whenever referring to differences between women and men. Thus, the term gender will be used throughout this report to encompass a wide range of behaviors, attitudes and personality traits associated with being a woman or a man.
Traditional gender roles are believed to contribute to women's greater vulnerability for anxiety and phobias (Fodor, 1974). Fodor argued that this predisposition was due to gender socialization teaching women to be dependent, fearful, passive, and submissive, whereas men are taught to be courageous, active, and goal and achievement oriented. Although research has consistently demonstrated a relationship between self-reported fear and gender role traits, such as femininity and masculinity, there is less consensus about which gender role contributes to anxiety and fear. Some studies have suggested that femininity was related to elevated fear of harmless animals (Dillon, Wolf, \& Katz, 1985; Tucker \& Bond, 1997), while others have demonstrated that identifying with a more masculine gender role was negatively associated with fear and anxiety (Arrindell, 2000; Chambless \& Mason, 1986). For example, low masculinity (e.g., lack of assertiveness) was associated with anxiety and avoidant behavior, whereas high femininity (e.g., nurturing and kind) was not associated with avoidance and anxiety (Chambless \& Mason, 1986). This inconsistent pattern has also been observed in children. While Ginsburg and Silverman (2000) reported that low masculinity was associated with number of fears endorsed among children, others have demonstrated that high femininity was related to anxiousness in children (Muris, Meesters, \& Knoops, 2005).

This well-established gender effect also might be explained by differential reinforcement boys and girls receive from parents and society. Since it is acceptable for girls to express anxiety and sadness, these emotions tend to be encouraged in girls, whereas boys are expected to act courageously and face their fears (Bem, 1981) perhaps providing boys with more opportunity for fear reduction. An example of parents encouraging gender-conforming behaviors in their children is demonstrated in a study conducted by Stevenson-Hinde and Shouldice (1993). In their study, parents approved of their girls' shyness and disapproved of the same shy behaviors if portrayed by boys. In fact, parents became less accepting of shy behaviors the older the boy was. This differential reinforcement is observed in the school settings as well. Buck (1975) demonstrated that teachers respond differently to the same behavior depending on the gender of the child. It was reported that teachers ignored girls' active involvement but provided positive attention when girls complied with cul- 
turally accepted gender roles, such as being submissive. Overall, boys received more positive reinforcement for being assertive, independent and active, while girls were rewarded for showing empathy and being social (e.g., Keenan \& Shaw, 1997).

Given that this differential reinforcement begins early in life and continues to be reinforced by society, it is possible that men and women may not be equally motivated to report their anxiety. One could speculate that men might be underreporting their actual fear or distress to be consistent with traditional masculine gender roles and avoid being perceived as vulnerable or weak, while it is more acceptable for women to express their true fears without fearing negative consequences (Craske, 2003; McLean \& Anderson, 2009). Pierce and Kirkpatrick (1992) tested whether men underreport their fear levels by asking participants to complete the same fear survey on two separate occasions. When participants came in for the second session, they were being informed that their answers could be "verified" by measuring their heart rate while they viewed images related to the items on the questionnaire. The authors demonstrated that, by making participants believe that their truthfulness was being verified, men showed significantly higher ratings during the second session compared to responses they provided at the initial meeting, while women's responses did not differ between the sessions. While the study demonstrated that men underreport their fear on self report measures, this reporting bias did not completely account for the gender effect as women still reported higher anxiety levels than men during the second session (Pierce \& Kirkpatrick, 1992).

To further explore the response bias hypothesis, Egloff and Schmukle (2004) examined participant's responses to both explicit (self-report measures) and implicit (Implicit Association Test and the Emotional Stroop Task) measures of anxiety. Implicit measures are believed to allow for a better exploration of whether or not the gender effect is due to response bias, since they allow for an indirect way of measuring anxiety. Egloff and Schmukle (2004) demonstrated that women reported greater anxiety than men on both implicit and explicit tests. However, the effect size on the implicit measures was half the size of that for the explicit measures.

While research has consistently shown that women report greater fear and anxiety on self-report measures (see McLean \& Anderson, 2009 for review), and tend to display more anxiety during stressful tasks (e.g., Chaplin, Hong, Bergquist, \& Sinha, 2008; Kelly, Forsyth, \& Karekla, 2006), findings about the gender effect on physiological reactivity are rather conflicting. Although there is some indication that women and men experience similar physiological reactivity, such as electrodernal reactivity and heart rate during several CO2 inhalations (Kelly et al., 2006), there is a greater body of literature suggesting that women are physiologically more reactive to a stressful situation than men. Specifically, Kudielka, Buske-Kirschbaum, Hellhammer, and Kirschbaum (2004) reported that women displayed more elevated heart rate response during a stressful task (Trier Social Stress Test) than men. The same pattern of physiological reactivity was demonstrated in an adolescent sample. Anderson and Hope (2009) examined response patterns of socially anxious and non-anxious adolescents during a social situation and found that, regardless of social anxiety, girls had higher heart rate reactivity than boys during the speech. Further, Schmaus, Laubmeier, Boquiren, Herzer, and Zakowski (2008) examined the gender effect on repeated stressor as a measure of habituation. Their results revealed that watching a 7-min Holocaust video on two separate occasions (2 days apart) resulted in an elevated heart rate during the second presentation among women only (Schmaus et al., 2008). While the study suggested that women's greater risk for anxiety disorders may be due to lack of habituation, avoidance behavior during the video was not assessed, which may have contributed to the observed lack of habituation.

The gender effect has also been observed in avoidance behavior, such that anxious women displayed more avoidance than anxious men (e.g., Cameron \& Hill, 1989; Thyer, Himle, Curtis, Cameron, \& Nesse, 1985). Speltz and Bernstein (1976) examined avoidance behavior among college students who were fearful of snakes. Men, who reported high anxiety and fear, got closer to the snake than did highly fearful women. Behavioral observations indicated that both men and women appeared equally distressed during the approach task, suggesting that the observed gender effect in the study might be a result of subjective fear and avoidance. Thus, despite similar levels of fear and distress, men approached the feared object more than did women. Avoidance behavior has been reinforced in girls by society from an early age, while boys have been encouraged to face their fears and control anxiety (see Craske, 2003 for review). This continuous exposure likely allows boys to acquire corrective learning, disconfirming their initial fear to the feared situation or stimuli, which is the main principle of exposure therapy (e.g., Barlow, 2004; Craske, 1999). The culturally reinforced coping strategy for girl on the other hand, is to avoid or escape from fear-inducing situations, interfering with their opportunity to overcome their fears.

The current study follows a previous project in our laboratory which attempted to replicate Pierce and Kirkpatrick's (1992) findings and investigate whether men would report higher subjective fear levels during the task if they were told that their fear ratings would be verified with their heart rate data, as opposed to those who received instructions that the heart rate was not a good measure of fear (McLean \& Hope, 2010). Participants were randomly assigned to one of two heart rate conditions (verifiable and irrelevant) prior to attempting the Behavioral Approach Test (BAT). The BAT consisted of 12 steps that culminated in touching the spider for $3 \mathrm{~s}$ with 2 fingers. Unlike Pierce and Kirkpatrick's (1992) which found men had a tendency to underreport their anxiety, there was no difference in men's reports, regardless of whether they believed their anxiety level could be verified by a heart rate monitor. Other results indicated that greater fear of spiders was associated with greater subjective anxiety and more avoidance during the BAT. Consistent with results from community and clinical samples, women reported greater anticipatory and peak anxiety ratings and demonstrated more avoidance of the feared object than men. Regardless of gender, low instrumentality, or low masculinity, was associated with more avoidance. Interestingly, greater expressivity, or the identification with a feminine gender role, was associated with more avoidance among men only, but was not related to avoidance or fear among women. However, given that the BAT demonstrated a ceiling effect, it is possible that the study was not able to accurately measure the relationship between gender role and avoidance. Furthermore, there was some indication that participants might have felt pressured to complete the task in order to receive study credits, which may have contributed to the high completion of the BAT.

In sum, prior research has consistently demonstrated that women report greater anxiety and fear in both clinical and community samples. Gender socialization practices and cultural expectations provide one possible explanation for the gender effect, socializing children from a young age to conform to gender-specific traits. While the literature has provided support for the importance of gender roles in understanding women's greater risk for anxiety disorders, the results remain mixed regarding which specific gender role traits account for the gender effect. While some studies have demonstrated that high expressivity (femininity) was related to greater fear, others have shown that low instrumentality (masculinity) was associated with more anxiety. Given the inconsistent findings and the fact that most studies have relied on self-report measure, with only a few utilizing behavioral measures to aid in the explanation of the gender effect, it would be important to employ a more comprehensive assessment to further explore the gender role theory as it relates to anxiety.

The first goal of the current study was to again attempt to replicate Pierce and Kirkpatrick's (1992) findings that men, who are told their fear ratings will be verified with their heart rate data, report higher subjective fear than those who receive instructions that heart rate is not a good measure of fear. Most studies examining the gender effect in anxiety have relied primarily on self-reported fear, with only a few studies exploring avoidance behavior or physiological arousal. Thus the second goal of the present study was to examine the gen- 
der effect across multiple domains-subjective anxiety, heart rate reactivity and behavioral avoidance during an anxiety-provoking behavioral task and on self-report questionnaires. As with previous research, it was expected that women would report higher fear on selfreport questionnaires and during a behavioral task, demonstrate greater behavioral avoidance (McLean \& Hope, 2010) and experience greater heart rate reactivity when exposed to laboratory stressors (Kudielka et al., 2004; Schmaus et al., 2008). Given the potential importance of behavioral avoidance, exploratory analyses were also conducted to compare the experience of men and women who avoided or did not avoid touching the tarantula. Finally, based on gender socialization theories, it was hypothesized that high expressive traits would be associated with greater subjective anxiety during the behavioral task but high instrumentality would be associated with lower subjective anxiety and less avoidance.

\section{Method}

\subsection{Design}

The study employed a post-test only, independent groups design to examine predictors of reported fear levels and behavioral avoidance during a task designed to induce anxiety. After completing a battery of questionnaires, participants were asked to approach a live tarantula by following a series of steps (see Table 1) that became increasingly difficult and to report their anxiety levels during various time points.

\subsection{Participants}

Participants were 144 undergraduate students from the University of Nebraska-Lincoln (67 women and 77 men), with a mean age of $20.2(\mathrm{SD}=2.43)$. The sample represented all years of college: $41.7 \%$ Freshman, $17.4 \%$ Sophomore, 18\% Junior, 22.2\% Senior, and $0.7 \%$ Graduate School. In regards to ethnic background, the majority of participants were European American (82.6\%), 4.9\% were Asian American, 3.5\% were African American, 3.5\% were Hispanic, $0.7 \%$ were Middle Eastern, and 4.9\% identified as "other." All participants received psychology research credit for their participation. Those who were not 19 years of age at the time of their participation were asked to bring in a signed parental consent form. Also, when participants signed up to participate, they were unaware that the study involved a live tarantula to reduce selection and response bias on measures. After being informed of the spider, participants were informed that they have the opportunity to withdraw.

\subsection{Measures}

\subsubsection{Self-report measures}

The Fear of Spiders Questionnaire (FSQ; Szymanski \& O'Donohue, 1995) is an 18-item questionnaire that asks respondents to indicate their fear and avoidance of spiders, using an 8-point scale that ranges from 0 (totally disagree) to 7 (totally agree). Higher scores on the measure reflect higher level of spider fear. The FSQ has demonstrated excellent internal consistency (0.92; Szymanski \& O'Donohue, 1995), high test-retest reliability, $r=0.91$ (Muris \& Merckelbach, 1996), and adequate convergent validity with the Spider Phobia Questionnaire (SPQ; Klorman, Weerts, Hastings, Melamed, \& Lang, 1974). The SPQ has been selected to measure fear and avoidance of spiders. Chronbach's alpha was 0.96 for the present sample indicating excellent internal reliability.

The Personal Attributes Questionnaire (PAQ; Spence, Helmreich, \& Stapp, 1975) assesses gender roles by asking participants to rate their characteristics on a 5-point scale by presenting an item and its opposite (e.g., very passive-very active), or an item with its negative connotation (e.g., very competitive-not at all competitive). The PAQ comprises of three subscales: masculinity score $(\mathrm{M})$, femininity score $(\mathrm{F})$,
Table 1

Steps on the behavioral approach test.

\begin{tabular}{|c|c|}
\hline & Approach spider rapidly \\
\hline & Look down at spider in the closed terrarium \\
\hline & Remove the lid of the terrarium \\
\hline & Place hands on both sides of the terrarium (indicated by a mark) \\
\hline & Remove the small container over spider \\
\hline & $\begin{array}{l}\text { Place palm of hand on the floor of the terrarium (across from } \\
\text { where spider is) }\end{array}$ \\
\hline & Gently touch spider with one finger for $5 \mathrm{~s}$ \\
\hline & Gently touch the spider for $10 \mathrm{~s}$ with 3 fingers \\
\hline & Allow spider to walk on bare hand for $30 \mathrm{~s}$ \\
\hline
\end{tabular}

and a masculinity-femininity score (M-F). The M scale assesses stereotypically masculine qualities such as competitiveness and independence also referred to as instrumental behaviors, while the $\mathrm{F}$ scale assesses stereotypically feminine qualities such as warmth and affection for others, also known as expressive traits (Spence \& Helm-

reich, 1978). The PAQ has demonstrated adequate internal consistency, $a=0.75-0.85$ (Toller, Suter, \& Trautman, 2004; Wilson \& Cook, 1984), and adequate test-retest reliability. The PAQ is included as a measure of gender roles. For the current study, Cronbach's alphas were 0.74 for the PAQ-F and 0.64 for the PAQ-M, indicating an adequate internal consistency for the PAQ-F, and questionable internal consistency for PAQ-M.

\subsubsection{Behavioral measure}

A behavioral approach test (BAT) was used to allow for an objective measure of participants' avoidant behavior when confronted with an anxiety-provoking stimulus. The steps on the BAT were modeled after those used in McLean and Hope (2010) and were slightly modified to make the task more challenging, given the ceiling effects in the last study. Participants were asked to approach a live tarantula in a graded step fashion: entering the testing room and approaching the spider rapidly (step 1); looking down at the spider in the closed terrarium (step 2); removing the lid of the terrarium (step 3); placing both hands on both sides of the terrarium (step 4) which required them to lean forward; removing the smaller container placed over the spider (step 5); placing the palm of their hand on the floor of the terrarium for $10 \mathrm{~s}$ across from where the spider was located (step 6); gently touching the spider with one finger for $5 \mathrm{~s}$ (step 7); gently touching the spider with three fingers for $10 \mathrm{~s}$ (step8); and allowing the spider to crawl on their hand for a duration of $30 \mathrm{~s}$ (step 9). Avoidance was measured on a 9 point scale, ranging from 0 (completely avoided BAT) to 9 (fully completed BAT).

2.3.2.1. Subjective anxiety. Participants were asked to report their subjective fear levels at various time points during the task using the Subjective Units of Distress scale (SUDS; Wolpe, 1973), which is a 100-point scale, where $0=$ no fear, $25=$ mild fear, $50=$ moderate fear, $75=$ severe fear, and $100=$ very severe fear. Participants indicated their anxiety prior to the BAT (anticipatory), after the initial step as they were standing in front of the tarantula (approach), at the end of the BAT (end) and were asked to recall when their anxiety level was the highest (peak).

2.3.2.2. Physiological reactivity. Heart rate (beats per minute) was recorded continuously throughout the BAT using a Polar S610i heart rate monitor. This monitor provides wireless heart rate monitoring with ECG-accurate continuous measurement, sampling once every five seconds. It consists of an elastic belt that attaches around the participant's chest and a wrist-watch receiver that records the heart rate data. Following a 2-min adaptation period, a 5-min resting heart rate was used to calculate heart rate reactivity for every minute (i.e., the difference in mean heart rate during entire 5 min resting and mean minute-by-minute heart rate during the BAT). 


\subsection{Procedures}

The study consisted of two parts, conducted one after the other, to ensure that participants' responses to the questions would not be biased based on the upcoming task. All procedures were approved by the Institutional Review Board at the University of NebraskaLincoln. For the first part, participants completed an informed consent form after which they were given a battery of questionnaires (FSQ, PAQ, and some other measures not reported here) that also included demographic information, such as age, gender, ethnicity, and year in college.

While participants completed the questionnaire packet, the experimenter randomly assigned them to one of two conditions:

Verifiable Heart Rate (verifiable HR) or Irrelevant Heart Rate (irrelevant HR). Then, participants were shown an introductory video on the computer that introduced the tarantula for this study. Participants listened to a description of the second part of the study and were asked to complete the second consent form at that point. Participants watched a demonstration on how to place the heart rate monitor and were shown to the bathroom to attach it and wash their hands with unscented soap before returning to the room. Once they returned, the experimenter started the monitoring equipment and presented the manipulation video on the computer. Depending on the assigned condition, participants received different information about the purpose of the heart rate monitor. Those in the verifiable HR condition listened to the following information on the computer:

"During the course of the study your heart rate will be monitored so that we can measure your true level of anxious arousal. Research has consistently shown that heart rate is a reliable measure of fear and anxiety. Therefore, heart rate is one of the measures used in a lie detector test because it changes with your emotions. This makes it important to rate your fear levels throughout the experiment as well as the items on the questionnaire as accurately as possible because we will compare your answers with changes in your heart rate."

Participants assigned to the irrelevant HR condition heard the following information:

"During the course of the study your heart rate will be monitored so we can test the equipment. Research has consistently shown that heart rate is an unreliable measure of true anxiety levels. In addition, this particular monitoring device has not been reliable in previous studies. The general consensus is that heart rate changes don't necessarily correspond with true level of fear. Therefore we don't expect your heart rate data to match up with your reported ratings of anxiety."

After the manipulation video clip, participants were asked to summarize the information provided in the video and then asked to fill out the FSQ again. Participants were then familiarized with the 0-100 SUD scale and given examples to illustrate the scale. Heart rate was recorded for a 5-min adjustment period during which participants were left alone in the room and were instructed to remain standing to avoid postural changes.

Following the adjustment period, the experimenter was replaced with another one who was blind to the heart rate condition. Then, participants watched a detailed description of every step they would be asked to complete during the BAT and were reminded that they could discontinue the task when they felt uncomfortable proceeding. After the steps were described, participants were asked to write down their SUDS rating on a confidential sheet of paper and place it in the box. Then, the experimenter informed participants of the first step, which entailed entering the testing room and rapidly approaching the tarantula sitting on the table across the room. Throughout the BAT, the experimenter observed the participant behind a one-way mirror and provided instructions for each subsequent step through an intercom system. As soon as the participant reached the table with the tarantula (step 1), they were asked to indicate their SUDS level by circling a number on a SUD scale (referred to as approach SUDS from here on). Prior to touching the spider (step 7) and prior to allowing the spider on their hand (step 9), participants were reminded that they could stop the task and were asked whether they would like to proceed. After completing the BAT, participants were asked to indicate their SUDS rating at the end of the task as well as provide their anxiety level at its peak. Participants who stopped prematurely were asked to indicate their anxiety level when they stopped as well as the highest it reached (peak). At the end of the BAT, participants removed the monitoring equipment and washed their hands with soap. When the participant returned, they were asked to fill out a feedback sheet sharing their experiences about the task and wearing the heart rate equipment. At the end, participants watched a debriefing video on the computer that provided more information about tarantulas and informed them of the actual purpose of the heart rate monitor.

All study procedures were presented on a computer to standardize the delivery of information and prevent any potential deviations from a written script. Further, the experimenters followed a procedures manual to ensure that the delivery of the videos and additional information was consistent across experimenters. Five research assistants received extensive training with the research protocol. Training sessions involved observation of a trained experimenter, and several practice sessions until experimenters reached proficiency in procedures. Experimenters were provided with a verbatim script of answers to common questions participants might ask, which were adapted from McLean and Hope (2010).

The phobic stimulus used in this study was a nonpoisonous Chilean rose-haired tarantula (Grammostola rosea; leg span approximately $8 \mathrm{~cm}$ ). This species has been widely used for fear experiments because of its intimidating appearance and lack of real danger to participants. In fact, Chilean rose tarantulas are known to be rather docile, calm and slow moving (Webb, 1992).

\section{Results}

\subsection{Attrition}

Of 171 participants, 86 were randomized to the verifiable HR condition, and 85 were randomized to the irrelevant HR condition. Eight participants $(4.7 \%)$ were excluded from data analyses due to technical problems resulting in missing data. They did not differ on any demographic or criterion measures from those who completed the study ( $\left.\mathrm{p}^{\prime} \mathrm{s}<0.05\right)$. Additionally, 17 participants $(9.8 \%)$ were excluded due to prior experience with tarantulas. Preliminary analyses revealed that these 17 participants had lower ratings than the rest of the group on subjective anxiety (anticipatory SUDS, $M=16.29$ vs. $M$ $=29.03, F(1,167)=7.7, p<0.01$; peak SUDS, $M=36.63$ vs. $M=56.62$, $F(1,161)=9.57, p<0.01$; end of task SUDS, $M=18.88$ vs. $M=39.21$, $F(1,162)=8.64, p<0.01)$, and FSQ $(M=28.53$ vs. $M=41.98, F(1,169)$ $=6.38, p<0.05$ ).

To examine the effectiveness of the manipulation, participants were asked to indicate on an open-ended questionnaire what they believed was the purpose of the heart rate monitor. Their responses were examined to determine if they believed the rationale provided. All but two participants provided a response that was in line with the provided rationale. The two participants $(1.2 \%)$ were excluded from further analyses because they clearly doubted the manipulation. There were no differences between the two excluded subjects and the rest of the participants on any demographic information or criterion variables. Our final sample consisted of 144 participants, 74 in the verifiable heart rate condition, and 70 in the irrelevant heart rate condition.

\subsection{Replication of response bias study}

Preliminary analyses revealed that the verifiable HR and irrelevant HR groups did not differ on demographic variables, such as gender $\left(X^{2}=1.31, p=0.25\right)$, age, $F(1,142)=0.08, p=0.78$, ethnicity $\left(X^{2}=5.47\right.$, 
$p=0.36)$, or year in college $\left(X^{2}=3.14, p=0.53\right)$. Also, the two conditions did not significantly differ on gender related measure, such as PAQ-F, $F(1,141)=0.197, p=0.66$, and PAQ-M, $F(1,141)=1.63, p=0.20$. To examine differences between the two HR groups in regards to subjective fear measures, two mixed-factorial ANOVAs were conducted with group and gender as the between-groups factors and self-reported fear (FSQ and SUDS) as the dependent variables. A 2 $($ HR group: verifiable, irrelevant) $\times 2$ (Time: pre and post manipulation FSQ) $\times 2$ (Gender: men and women) mixed-factorial ANOVA was used to test the hypothesis that men in the verifiable heart rate condition would score higher on the FSQ measure at the second administration compared to the first administration, while this observation would not be observed for women. Contrary to our hypothesis, the effect of HR group was not significant, $F(1,136)=1.605, p$ $=$ ns. There was a main effect of gender, $F(1,136)=18.7, p<0.001$, with women scoring higher on the FSQ compared to men (see Table 2 ), and a main effect of time, $F(1,136)=39.228, p<0.001$, indicating that regardless of HR group or gender, participants reported higher scores on the FSQ the second time it was administered. Further, there was no gender $\times$ time interaction, $F(1,136)=1.07, p=\mathrm{ns}$, and no gender $\times$ time $\times$ group interaction, $F(1,136)=2.56, p=$ ns.

The second 2 (HR group: verifiable, irrelevant) $\times 2$ (Gender: men and women $) \times 4$ (SUDS: anticipatory, approach, peak, and end) mixed-factorial ANOVA was computed to test whether men would report greater subjective anxiety in the verifiable HR group than in the irrelevant HR group. Results indicated no main effect of HR group, $F(1,135)=0.48, p=n$ s, such that information received about the heart rate monitor did not affect subjective anxiety. There was a main effect of gender, $F(1,135)=9.30, p<0.005$. Follow-up LSD analyses revealed that women displayed greater SUDS than men during the BAT (see Table 2). Also, a main effect of time was observed $($ Wilks $=0.28, F(3,133)=115.53, p<0.001)$. Follow-up LSD analyses indicated that subjective anxiety ratings during the BAT were higher than in anticipation of the task (see Figure 1). There was a significant decrease in SUDS from peak to end of task reports. Furthermore, the interaction between time and gender as it relates to subjective anxiety was significant (Wilks $=0.89, F(3,133)=5.18, p<0.005)$. Followup LSD analyses revealed that the gender effect described above was only present during the BAT, while there was no difference between men and women on anticipatory SUDS (see Table 2). Also, a closer examination of the interaction revealed that end of task SUDS were higher than approach SUDS for women only, while that difference was not significant for men. There was not a multivariate interaction between time and HR group (Wilks $=0.99, F(3,133)=0.48, p=n s$ ), or a multivariate interaction between gender and HR group, $F(1,135)=$ $1.63, p=n s$, or a multivariate interaction between time, gender, and group $($ Wilks $=0.99, F(3,133)=0.08, p=n s)$.

\subsection{Gender effect across multiple domains}

Preliminary analyses were conducted to examine whether men and women differed on demographic variables, demonstrating that there were no significant differences between men and women related to age, $F(1,142)=0.09, p=0.76$ and ethnicity, $X^{2}=2.02, p=0.85$. As shown in the previous section, there was a gender effect on FSQ and SUDS, with women scoring higher on the FSQ and SUDS during the BAT. Additionally, men and women did not differ on PAQ$\mathrm{F}, F(1,141)=3.72, p=\mathrm{ns}$, but there was a gender effect on PAQ-M, $F(1,141)=10.09, p<0.005$, with men scoring higher on the instrumentality measure than women (see Table 2).

\subsubsection{Heart rate reactivity}

To further explore the gender effect during the BAT, a mixed group factorial ANOVA was used to examine objective physiological arousal by gender. Heart rate data was limited to the first 2-min of the BAT (i.e., prior to touching spider) due to loss of available data afterwards. Additionally, heart rate data was only available for 125

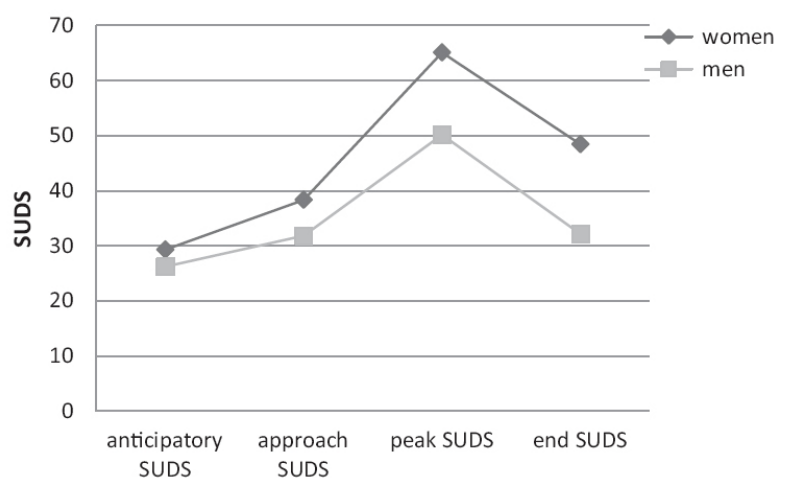

Figure 1. Subjective Units of Distress (SUDS) during BAT by gender.

participants due to technical malfunction with the heart rate equipment. Gender was the between-groups factors, while heart rate reactivity during the first $2 \mathrm{~min}$ of the BAT was a repeated measure. Contrary to our expectations, the 2 (Gender: men and women) $\times 2$ (Time: $\mathrm{HR}$ reactivity for first and second minute of BAT: mean baseline subtracted from each minute of BAT) mixed factorial ANOVA revealed no main effect for gender, $F(1,123)=2.52, p=$ ns. There was a main effect for time, Wilks $=0.671, F(1,123)=60.32, p<0.001$, in that a significant drop in heart rate reactivity was observed between the first and second minute of the BAT, and no interaction between gender and time, Wilks $=0.99, F(1,123)=1.52, p=$ ns.

\subsubsection{Avoidance}

In regards to avoidance, women completed significantly fewer steps on the BAT, $F(1,141)=27.81, p<0.001$, suggesting that women were more likely to terminate the BAT prematurely and displayed greater avoidance (see Table 2 and Figure 2). Further analyses revealed that significantly fewer women (31\%) touched the spider $\left(X^{2}=12.68, p<\right.$ $0.001)$ than men (61\%). Fewer women (18\%) chose to have the spider crawl on their hand $\left(X^{2}=21.84, p<0.001\right)$ compared to men $(56 \%)$.

Given the above mentioned gender effect, we were interested in further exploring what might contribute to avoidance. Two between groups factorial ANOVAs were conducted to examining heart rate reactivity and subjective anxiety between men and women who touched the spider versus those who did not. Thus, avoidance in these analyses was operationalized as whether or not a participant touched the spider. A 2 (Gender: men and women) $\times 2$ (Touch: whether or not touched spider) between groups factorial ANOVA was used, with gender and touch of spider being the betweengroups factors and heart rate reactivity during the second minute serving as the dependent variable. While there was no main effect of gender, $F(1,121)=0.39, p=$ ns or avoidance, $F(1,121)=0.396, p=$ $\mathrm{ns}$, the model revealed an interaction between gender and avoidance of the tarantula as they relate to heart rate reactivity during the second minute of the BAT, $F(1,121)=4.92, p=0.028$. Follow-up LSD analyses of the cell means (minimum mean difference $=3.327$ ) revealed that among participants who did not touch the spider, women $(M=3.41, \mathrm{SD}=7.13)$ displayed greater heart rate reactivity than men $(M=-0.20, \mathrm{SD}=6.85), t=-2.043, d f=63, p=0.04$, but women $(M=-0.2, \mathrm{SD}=5.34)$ did not differ from men $(M=1.81, \mathrm{SD}=6.51)$ on heart rate reactivity if they did touch the spider, $t=1.13, d f=58$, $p=$ ns (see Figure 3 ).

The second 2 (Gender: men and women) x 2 (Touch: whether or not touched spider) between groups factorial ANOVAs was computed, with gender and touch of spider being the between-groups factors 


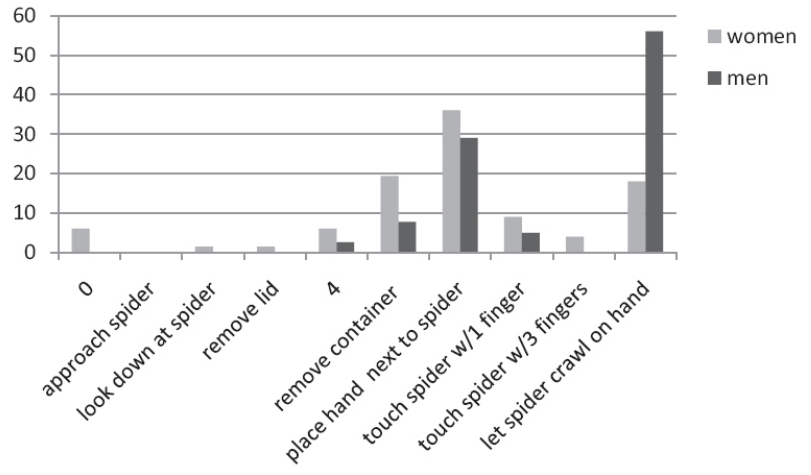

Figure 2. Percent of participants ending the BAT at each step by gender

and peak SUDS serving as the dependent variable. Results revealed a main effect of gender, $F(1,121)=7.5, p<0.01$, with women reporting higher peak SUDS, a main effect of avoidance, $F(1,121)=21.2, p$ $<0.001$, such that participants who avoided touching the spider reported greater peak SUDS, and a significant interaction, $F(1,121)=$ $5.37, p<0.05$. Follow-up analyses demonstrated that women $(M=$ $68, \mathrm{SD}=21.3)$ and men $(M=66.3, \mathrm{SD}=21.2)$ who did not touch the tarantula did not differ on peak SUDS $(t=-0.32, d f=63, p=n s)$, while there was a gender effect among participants who touched the spi$\operatorname{der}(t=-3.13, d f=58, p<0.01)$, with women $(M=59, \mathrm{SD}=24)$ reporting higher peak SUDS than men $(M=38.5, \mathrm{SD}=19.7)$ as can be seen in Figure 3.

\subsection{Gender role analyses}

Correlational analyses were used to examine the relationship between gender-role (PAQ-F, PAQ-M), and subjective anxiety experienced (anticipatory SUDS, approach SUDS, end of task SUDS, and peak SUDS), and number of steps completed during the BAT. Surprisingly, the PAQ-F was not significantly correlated with any of the criterion measures. However, the PAQ-M was negatively correlated with peak SUDS, $r(141)=-0.22$, and end of task SUDS, $r(141)=-0.17$, revealing that participants with high instrumentality reported lower peak anxiety and less anxiety at the end of the behavioral task. Although, the PAQ-M was correlated with peak SUDS for women, $r(64)=-0.26, p<0.05$, and not for men, $r(75)=-0.06$, this difference was not significant $(Z=1.20, p=n s)$. Furthermore, the PAQ-M was

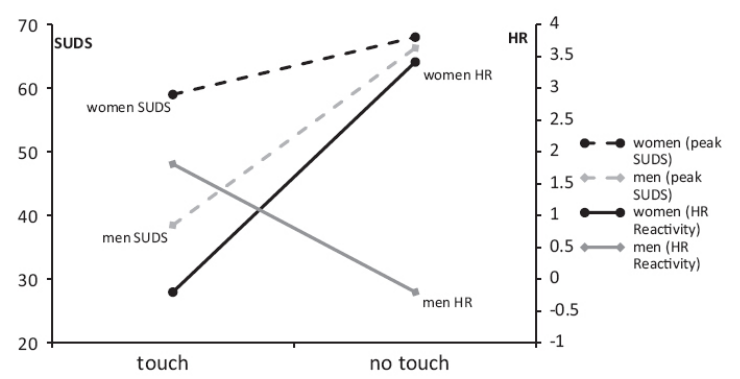

Figure 3. Concordance among behavioral, physiological, and subjective anxiety. Note: SUDS, Subjective Units of Distress, peak anxiety rating; HR, heart rate reactivity during minute 2 of BAT. significantly correlated with anticipatory SUDS for women, $r(64)=$ $-0.29, p<0.05$, but not for men, $r(75)=0.15$, suggesting that women with high instrumentality reported less anticipatory anxiety. Further analyses revealed that this difference was statistically significant $Z=$ $2.63, p<0.01$. The PAQ-M was not correlated with BAT steps, $r(141)$ $=0.15, p=$ ns.

\section{Discussion}

Despite the well established gender effect in anxiety disorders, our understanding about the nature of this effect is limited. The study investigated the effect of gender on self-reported anxiety, willingness to report fear, avoidance, and physiological arousal.

To evaluate the effect of perceived confirmability on self-report and subjective fear, we utilized a combination of Pierce and Kirkpatrick's (1992) and McLean and Hope's (2010) methods. In particular, we had participants complete the FSQ on two separate occasions, once before knowing what the rest of the study entailed and once after being informed about the second part of the study. Secondly, similar to McLean and Hope (2010) we examined the effect gender and HR group had on subjective fear reported during the BAT. Similar to McLean and Hope (2010), we were unable to replicate Pierce and Kirkpatrick's (1992) findings that men would underreport their fear when informed that their responses could be verified by heart rate. Surprisingly, we did find an effect of time, such that all participants regardless of group reported higher fear scores on the FSQ the second time it was administered. It is possible that watching an in-

Table 2

Means and standard deviation on study measures by gender.

\begin{tabular}{|c|c|c|c|c|c|c|c|c|}
\hline \multirow[t]{2}{*}{ Variable } & \multicolumn{3}{|c|}{ Women $(n=66)$} & \multicolumn{4}{|c|}{$\operatorname{Men}(n=77)$} & \multirow[b]{2}{*}{ Cohen's $d$} \\
\hline & $M$ & SD & Range & $M$ & SD & Range & $F$ & \\
\hline BAT & 5.90 & 2.20 & $0-9$ & 7.60 & 1.66 & $4-9$ & $27.81^{* * *}$ & 0.87 \\
\hline FSQ & 49.68 & 22.35 & $18-95$ & 35.01 & 18.20 & $18-95$ & $18.71^{* * *}$ & 0.72 \\
\hline PAQ-F & 24.47 & 3.08 & $17-28$ & 23.42 & 3.40 & $17-28$ & 3.72 & 0.32 \\
\hline PAQ-M & 20.56 & 3.56 & $14-29$ & 22.49 & 3.69 & $14-29$ & $10.09^{* *}$ & 0.53 \\
\hline Antic SUDS & 31.95 & 20.08 & $0-100$ & 26.01 & 16.70 & $0-80$ & 3.73 & 0.32 \\
\hline End SUDS & 51.20 & 29.30 & $0-100$ & 31.94 & 25.54 & $0-90$ & $17.64^{* * *}$ & 0.71 \\
\hline Peak SUDS & 66.98 & 22.32 & $15-100$ & 49.96 & 24.52 & $10-95$ & $18.60^{* * *}$ & 0.73 \\
\hline
\end{tabular}

Note: Antic SUDS, Subjective Units of Distress, Anticipatory Anxiety Rating; BAT, Behavioral Approach Test (total number of steps completed, 0-9); End SUDS, Subjective Units of Distress, End of Task Anxiety Rating; FSQ, Fear of Spider Questionnaire Total Score; PAQ-F, Personal Attributes Questionnaire, Feminine Subscale; PAQ-M, Personal Attributes Questionnaire, Masculine Subscale; Peak SUDS, Subjective Units of Distress, Peak Anxiety Rating. $* * p<0.01$.

$* * * 0<0.001$. 
troductory video of the study tarantula and a mock participant complete the BAT might have served as a prime by making the phobic stimulus of the spider more salient.

Given the failure to replicate Pierce and Kirkpatrick (1992) again, we explored the manipulation check in more detail to see if it was not sufficiently salient. We identified 38 participants, who gave some indication that heart rate is related to fear in open-ended questions, with 32 being in the irrelevant HR condition and 6 in the verifiable HR condition. This suggests that the information provided was not as effective as we would have liked in generating the irrelevant HR condition. It is possible that participants might have become suspicious because they were asked to wear the heart rate monitor despite of being informed that heat rate was not a reliable measure of true anxiety. However, instead of excluding all potentially suspicious participants from the analyses, which would have significantly affected cell sizes and randomization, we decided to include them to preserve the randomization effect. As noted above, we did exclude two participants who clearly indicated they rejected the bogus explanation.

Our second goal was to examine the gender effect across several domains. Similar to previous findings in our laboratory (McLean \& Hope, 2010), women displayed more anxiety and avoidance of the fear-inducing task than men, which was demonstrated by their greater fear of spiders, greater peak and end of task anxiety, and greater avoidance on the BAT. There was no gender effect on heart rate reactivity. Unlike McLean and Hope (2010), the gender effect in the current study was not present for anticipatory anxiety. The modifications of our procedures could explain this difference in findings between the two studies. While participants in McLean and Hope's (2010) study were verbally informed about the specifics of the BAT, the current study included a brief video presenting the study tarantula prior to providing a description of the details. Thus, providing a visual of the anxiety-provoking stimulus may have increased participants' anxiety while anticipating the task. This is further confirmed by the increase in heart rate reactivity during the first minute of the BAT, suggesting that all participants regardless of gender displayed an elevated physiological arousal.

Given the importance of avoidance in anxiety, we explored avoidance and its correlates in some detail. Women avoided more by all measures-touching spider, letting spider crawl on hand.More interesting, as the task became more challenging the gender effect became greater such that even fewer women allowed the spider to crawl on their hand compared to men. This face valid comparison helped identify individuals who functioned differently in this standard situation and it is clear that men's and women's experience touching or not touching the spider differed. One would expect high concordance across the variables-behavioral avoidance (not touching the spider), high subjective anxiety, and high physiological arousal should co-occur. Interestingly, the concordance pattern differed by gender. Women were concordant for physiological arousal, and avoidance but reported high subjective anxiety regardless of whether they avoided, suggesting that it may be physiological arousal that drives women's behavior rather than subjective anxiety. Men were concordant across subjective anxiety and avoidance but reported higher physiological arousal when they did not avoid than when they did. Women who touched the spider said they felt anxious but this was not indicated by their heart rate which was near baseline. Men who touched the spider indicate low anxiety but had somewhat elevated heart rate. Our correlational data preclude causal statements regarding what factors might determine avoidance behavior. However, future research exploring this apparent differing experience in the face of feared stimuli may help elucidate the differences in prevalence of anxiey by gender.

One possible exploration could focus on examining Taylor et al.'s (2000) tend-and-befriend hypothesis in relation to avoidance. Specifically, they proposed that the fight-or-flight stress response may not be accurately depicting women's behavioral response to stressful situations. Instead, Taylor et al.'s (2000) proposed that women display behaviors that would protect and care for the offspring, and reduce stress. Thus, while the tending and befriending nature of response would be protective for the offspring, it could also contribute to greater avoidance of potentially threatening situations. Thus, befriending (or relying on support) may prevent women from developing a more independent coping style for potentially threatening situations.

Finally, our results provided partial support for our third hypothesis demonstrating that instrumental characteristics (PAQ-M) were negatively related to peak and end of task SUDS during the BAT. These results are consistent with findings from Chambless and Mason (1986) and Ginsburg and Silverman (2000), who concluded that higher scores on the masculinity measure were associated with less fear and anxiety among adults and children. Moreover, conforming to a more traditional masculine gender-role (i.e. instrumentality) was associated with lower anticipatory subjective anxiety for women only, suggests that it may have a buffering effect on subjective anxiety for women specifically. Experiencing lower anticipatory anxiety prior to a potentially fearful task may increase the likelihood that women will face their fears, allowing them to ultimately overcome their fears. Contrary to our expectations, we did not find a relationship between expressive traits (i.e., femininity) and anxiety or avoidance, which is inconsistent with previous work by Dillon et al. (1985) and Gallacher and Klieger (1995). Thus, our results suggest that lower instrumentality rather than greater expressivity traits are related to anxiety and reported fear of spiders.

While gender was related to the BAT, with women demonstrating greater avoidance, gender roles were not associated with behavioral avoidance. This finding was not consistent with McLean and Hope (2010), who demonstrated that greater instrumentality was related to less avoidant behavior regardless of gender. However, our inability to find a relationship between gender roles and avoidance behavior was similar to Gallacher and Klieger (1995). This suggests that while gender roles, in particular low instrumentality, was related to high anxiety experienced during a fear-inducing task, it did not display the same pattern with avoidance.

There are several limitations to our study that need to be considered. Specifically, it was unclear whether our manipulation was successful at generating different groups given the significant elevation in the self-report measure the second time it was administered. Future studies should utilize a stronger manipulation, preferably using a different strategy to elicit truthful responses, since relying on heart rate equipment may have unintentionally tainted our manipulation. The inclusion of implicit measures of anxiety may further aid in the investigation of response bias.

Also, while this study is unique in examining the gender effect using subjective, physiological, and behavioral measures during an anxiety-provoking task, replication of the results is warranted. In addition, future studies should incorporate observational measures to explore whether participants' subjective experience can be verified by more objective measures. In particular, examining specific behaviors that have been associated with anxiety and fear could further test whether one's subjective experience matches particpants' presentation. Future studies should examine additional factors that may mediate the relationship between gender and avoidance, such as level of courageousness and explore their relationship to gender role orientation.

Another important limitation to our study is the limited generalizability of our results. Given that our sample consisted primarily of European American college students, it would be important for future research to examine the relationship between gender role and anxiety using a more ethnically diverse sample as well as explore whether similar patterns would be observed in a clinical sample. Another noteworthy shortcoming of our study is that we utilized self-report measures to assess gender role orientation. While questionnaires are very commonly used in assessing specific constructs, they often may not provide the most accurate information given the potential for biased responding. While there are 
no specific behavioral measures to our knowledge that assess gender role traits, a more objective and possibly comprehensive assessment may be called for. Furthermore, the lower than expected internal consistency of the PAQ, especially the PAQ-M, in our sample raises some questions about the reliability of the measure for our study. The limitations in measurement of gender roles may help explain the inconsistencies in findings about gender roles and anxiety in the literature. Even within our lab, we have inconsistent data across studies.

This is the second project from our laboratory that has not been able to replicate Pierce and Kirkpatrick's (1992) findings of underreporting of anxiety by men. Thus, our inability to replicate their results and the lack of any published studies to date confirming the response bias hypothesis for fear and anxiety, may suggest that this phenomenon may not be as universal and strong as originally suggested. It may be more fruitful to pursue other explanations for the gender effect in anxiety, several of which are suggested by findings in this study.

Overall, this study highlights that men and women appear to have quite different experiences when facing a potentially threatening stimulus. Future research should further examine these differences and their possible etiological and treatment implications.

\section{References}

Anderson, E.R., \& Hope, D.A. (2009). The relationship among social phobia, objective and perceived physiological reactivity, and anxiety sensitivity in an adolescent population. Journal of Anxiety Disorders 23: 18-26. DOI: 10.1016/j.janxdis.2008.03.011

Angst, J., \& Dobler-Mikola, A. (1985). The Zurich Study: V. Anxiety and phobia in young adults. European Archives of Psychiatry \& Neurological Sciences 235: 171-178. DOI: 10.1007/BF00380989

Arrindell, W.A. (2000). Phobic dimensions: IV. The structure of animal fears. Behaviour Research and Therapy 38: 509-530. DOI: 10.1016/ S0005-7967(99)00097-2

Arrindell, W.A., Eisemann, M., Richter, J., Oei, T.P.S., Caballo, V.E., vanderEnde, J., et al. (2003). Masculinity-femininity as a national characteristic and its relationship with national agoraphobic fear levels: Fodor's sex role hypothesis revitalized. Behaviour Research and Therapy 41: 795-807.

Barlow, D.H. (2004). Anxiety and Its Disorders: The Nature and Treatment of Anxiety and Panic ( $2^{\text {nd }}$ ed.). New York: Guilford Press.

Bem, S.L. (1981). Gender schema theory: a cognitive account of sex typing. Psychological Review 88: 354-364. DOI: 10.1037/0033295X.88.4.354

Bourdon, K.H., Boyd, J.H., Rae, D.S., Burns, B.J., Thompson, J.W., \& Lock, B.Z. (1988). Gender differences in phobias: results of the ECA community survey. Journal of Anxiety Disorders 2: 227-241. DOI: 10.1016/0887-6185(88)90004-9

Bruce, S.E., Yonkers, K.A., Otto, M.W., Eisen, J.L., Weisberg, R.B., Pagano, M., et al. (2005). Influence of psychiatric comorbidity on recovery and recurrence in generalized anxiety disorder, social phobia, and panic disorder: a 12-year prospective study. American Journal of Psychiatry 162: 1,179-1,187. DOI: 10.1176/appi.ajp.162.6.1179

Buck, R. (1975). Nonverbal communication of affect in children. Journal of Personality and Social Psychology 31: 644-653. DOI: 10.1037/ h0077071

Cameron, O.G., \& Hill, E.M. (1989). Women and anxiety. Psychiatric Clinics of North America 12: 175-186.

Chambless, D.L., \& Mason, J. (1986). Sex, sex-role stereotyping and agoraphobia. Behaviour Research and Therapy 24: 231-235. DOI: 10.1016/0005-7967(86)90098-7

Chaplin, T.M., Hong, K., Bergquist, K., \& Sinha, R. (2008). Gender differences in response to emotional stress: an assessment across subjective, behavioral, and physiological domains and relations to alcohol cravings. Alcoholism: Clinical and Experimental Research 32: 1,242-1,250. DOI: 10.1111/j.1530-0277.2008.00679.x
Craske, M.G. (1999). Anxiety Disorders: Psychological Approaches to Theory and Treatment. New York: Basic Books.

Craske, M.G. (2003). The Origins of Phobias and Anxiety Disorders: Why More Women than Men. Oxford, England: Elsevier Science.

Davey, G.C.L., McDonald, A.S., Hirisave, U., Prabhu, G.G., Iwawaki, S., Jim, C.I., et al. (1998). A cross-cultural study of animal fears. Behaviour Research and Therapy 36: 735-750. DOI: 10.1016/S00057967(98)00059-X

Dillon, K.M., Wolf, E., \& Katz, H. (1985). Sex roles, gender, and fear. Journal of Psychology 119: 355-359.

Egloff, B., \& Schmukle, S.C. (2004). Gender differences in implicit and explicit anxiety measures. Personality and Individual Differences 36: 1,807-1,815. DOI: $10.1016 / \mathrm{j} /$ paid.2003.07.002

Fodor, I.G. (1974). The phobic syndrome in women: implications for treatment. In: V. Franks, \& V. Burtle (Eds.), Women in Therapy: New Psychotherapies for a Changing Society. New York: Brunner/Mazel.

Gallacher, F., \& Klieger, D.M. (1995). Sex role orientation and fear. Journal of Psychology: Interdisciplinary and Applied 129: 41-49.

Ginsburg, G.S., \& Silverman, W.K. (2000). Gender role orientation and fearfulness in children with anxiety disorders. Journal of Anxiety Disorders 14: 57-67. DOI:10.1016/S0887-6185(99)00033-X

Keenan, K., \& Shaw, D. (1997). Developmental and social influences on young girls' early problem behavior. Psychological Bulletin 121: 95-113. DOI:10.1037/0033-2909.121.1.95

Kelly, M.M., Forsyth, J.P., \& Karekla, M. (2006). Sex differences in response to a panicogenic challenge procedure: an experimental evaluation of panic vulnerability in a non-clinical sample. Behaviour Research and Therapy 44: 1,421-1,430. DOI:10.1016/j.brat.2005.10.012

Kessler, R.C., Chiu, W.T., Demler, O., Merikangas, K., \& Walters, E.E. (2005). Prevalence, severity, and comorbidity of 12-month DSM-IV disorders in the National Comorbidity Survey Replication. Archives of General Psychiatry 62: 617-627. DOI:10.1001/archpsyc.62.6.617

Klorman, R., Weerts, T.C., Hastings, J.E., Melamed, B.G., \& Lang, P.J. (1974). Psychometric description of some specific-fear questionnaires. Behavior Therapy 5: 401-409. DOI: 10.1016/S00057894(74)80008-0

Kudielka, B.M., Buske-Kirschbaum, A., Hellhammer, D.H., \& Kirschbaum, C. (2004). Differential heart rate reactivity and recovery after psychosocial stress (TSST) in healthy children, younger adults, and elderly adults: the impact of age and gender. International Journal of Behavioral Medicine 11: 116-121. DOI: 10.1207/s15327558ijbm11028 McLean, C.P., \& Anderson, E.R. (2009). Brave men and timid women? A review of the gender differences in fear and anxiety. Clinical Psychology Review 29: 496-505. DOI: 10.1016/j.cpr.2009.05.003

McLean, C.P., \& Hope, D.A. (2010). Gender-role and behavioral avoidance: the influence of perceived confirmability of selfreport. Journal of Anxiety Disorders 24: 494-502. DOI: 10.1016/j. janxdis.2010.03.006

Muris, P., Meesters, C., \& Knoops, M. (2005). The relationship between gender role orientation and fear and anxiety in nonclinic-referred children. Journal of Clinical Child and Adolescent Psychology 34: 326-332. DOI: 10.1207/s15374424jccp340212

Muris, P., \& Merckelbach, H. (1996). A comparison of two spider fear questionnaires. Journal of Behavior Therapy and Experimental Psychiatry 27: 241-244. DOI: 10.1016/S0005-7916(96)00022-5

Pierce, K.A., \& Kirkpatrick, D.R. (1992). Do men lie on fear surveys? Behaviour Research and Therapy 30: 415-418. DOI: 10.1016/00057967(92)90055-L

Schmaus, B.J., Laubmeier, K.K., Boquiren, V.M., Herzer, M., \& Zakowski, S.G. (2008). Gender and stress: differential psychophysiological reactivity to stress reexposure in the laboratory. International Journal of Psychophysiology 69: 101-106. DOI: 10.1016/j.jpsycho.2008.03.006

Speltz, M., \& Bernstein, D.A. (1976). Sex differences in fearfulness: verbal report, overt avoidance, and demand characteristics. Journal of Behavior Therapy and Experimental Psychiatry 7: 117-122. DOI: 10.1016/0005-7916(76)90067-7 
Spence, J.T., \& Helmreich, R.L. (1978). Masculinity and Femininity: Their Psychological Dimensions, Correlates, and Antecedents. Austin, Tex.: University of Texas Press.

Spence, J.T., Helmreich, R.L., \& Stapp, J. (1975). Ratings of self and peers on sex role attributes and their relation to self-esteem and conceptions of masculinity and femininity. Journal of Personality and Social Psychology 32: 29-39. DOI: 10.1037/h0076857

Stevenson-Hinde, J., \& Shouldice, A. (1993). Wariness to strangers: a behavior systems perspective revisited. In: K.H. Rubin, \& J.B. Asendorpf (Eds.), Social Withdrawal, Inhibition, and Shyness in Childhood (pp. 101-116). New York: Lawrence Erlbaum.

Szymanski, J., \& O’Donohue, W. (1995). Fear of spiders questionnaire. Journal of Behavior Therapy and Experimental Psychiatry 26: 3134. DOI: 10.1016/0005-7916(94)00072-T

Taylor, S.E., Klein, L.C., Lewis, B.P., Gruenewald, T.L., Gurung, R.A.R., \& Updegraff, J.A. (2000). Biobehavioral responses to stress in females: tend-and-befriend, not fight-or-flight. Psychological Review 107: 411-429. DOI: 10.1037/0033-295X.107.3.411

Thyer, B.A., Himle, J., Curtis, G.C., Cameron, O.G., \& Nesse, R.M.
(1985). A comparison of panic disorder and agoraphobia with panic attacks. Comprehensive Psychiatry 26: 208-214. DOI: 10.1016/0010440X(85)90041-0

Toller, P.W., Suter, E.A., \& Trautman, T.C. (2004). Gender role identity and attitudes toward feminism. Sex Roles 51: 85-90. DOI: 10.10 23/B:SERS.0000032316.71165.45

Tucker, M., \& Bond, N.W. (1997). The roles of gender, sex role, and disgust in fear of animals. Personality and Individual Differences 22: 135-138. DOI:10.1016/S0191-8869(96)00168-7

Webb, A. (1992). The Proper Care of Tarantulas. Neptune City, N.J.: T.F.H. Publications.

Weissman, M.M., \& Merikangas, K.R. (1986). The epidemiology of anxiety and panic disorders: an update. Journal of Clinical Psychiatry 47 (Suppl.): 11-17.

Wilson, F.R., \& Cook, E.P. (1984). Concurrent validity of four androgyny instruments. Sex Roles 11: 813-837. DOI: 10.1007/ BF00287812

Wolpe, J. (1973). The Practice of Behavior Therapy. New York: Pergamon Press. 